\title{
Ferulic acid exerts antitumor activity and inhibits metastasis in breast cancer cells by regulating epithelial to mesenchymal transition
}

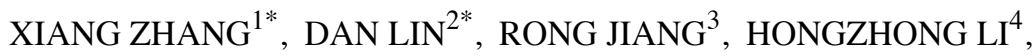 \\ JINGYUAN WAN ${ }^{2}$ and HONGYUAN LI ${ }^{1}$
}

\author{
${ }^{1}$ Department of Endocrine and Breast Surgery, The First Affiliated Hospital of Chongqing Medical University, \\ Chongqing 400016; ${ }^{2}$ Chongqing Key Laboratory of Biochemistry and Molecular Pharmacology, and \\ ${ }^{3}$ Laboratory of Stem Cell and Tissue Engineering, Chongqing Medical University, Chongqing 400016; \\ ${ }^{4}$ Molecular Oncology and Epigenetics Laboratory, The First Affiliated Hospital of \\ Chongqing Medical University, Chongqing 400016, P.R. China
}

Received January 23, 2016; Accepted March 9, 2016

DOI: $10.3892 /$ or.2016.4804

\begin{abstract}
Metastasis, which frequently occurs in breast cancer, is the major cause of mortality; therefore, new treatment strategies are urgently needed. Ferulic acid, isolated from Ferula foetida, a perennial herb, has shown antineoplastic activity in various types of cancers, such as colon and lung cancer, and central nervous system tumors. However, its potential role in suppressing breast cancer metastasis has not been fully understood. In the present study, we evaluated the antitumor activity of ferulic acid in breast cancer cell line-based in vitro and in vivo models. We first showed that ferulic acid treatment resulted in decreased viability, increased apoptosis and suppression of metastatic potential in breast cancer cell line MDA-MB-231. Furthermore, it was demonstrated that the antitumor activity of ferulic acid and its role in suppressing metastasis were regulated by the reversal of epithelial-mesenchymal transition (EMT). Consistent with our findings in vitro, the antitumor potential of ferulic acid was also verified in an MDA-MB-231 xenograft mouse model where significantly decreased tumor volume, weight and increased
\end{abstract}

Correspondence to: Professor Hongyuan Li, Department of Endocrine and Breast Surgery, The First Affiliated Hospital of Chongqing Medical University, 1 Youyi Road, Chongqing 400016, P.R. China

E-mail: hongy_li@hotmail.com

Professor Jingyuan Wan, Chongqing Key Laboratory of Biochemistry and Molecular Pharmacology, Chongqing Medical University, 1 Yixueyuan Road, Chongqing 400016, P.R. China

E-mail: jywan@cqmu.edu.cn

*Contributed equally

Key words: breast cancer, metastasis, ferulic acid, epithelialmesenchymal transition apoptosis were observed. Taken together, these results indicate that ferulic acid may be used as an effective therapeutic agent against breast cancer.

\section{Introduction}

Breast cancer is a systemic disease. It has become the most frequently diagnosed cancer and the leading cause of cancer-related death among females worldwide, with an estimated 1.7 million cases and 521,900 deaths in 2012 (1). There are several obstacles for breast cancer treatment; for example, chemoresistance, immune escape, metastasis and recurrence $(2,3)$. During these obstacles, metastasis is the leading cause of mortality in breast cancer patients. Nearly $50 \%$ of breast cancer patients treated with chemotherapeutic and/or hormonal agents develop distant metastatic disease $(4,5)$; these patients face a 5 -year survival rate of only $20 \%$ (6). Therefore, there is a great and urgent need to identify new drugs or treatments for metastatic breast cancer.

Tumor metastasis is a multi-step process by which tumor cells disseminate from their primary site and form secondary tumors at a distant site. In the past decade, a developmental process, epithelial-mesenchymal transition (EMT), has been increasingly recognized to play pivotal and intricate roles in promoting carcinoma invasion and metastasis (7). EMT programs were first observed in the context of embryonic development, where they function as transdifferentiation programs that effect critical morphogenetic steps, such as gastrulation and neural crest formation (8). Specifically, EMTs generate mesenchymal cell types from epithelial and endothelial precursors. These epithelial-mesenchymal conversions are crucial for cell movements that take place during breast cancer metastasis $(9,10)$. By imparting mesenchymal traits to carcinoma cells, an EMT can generate cellular traits associated with high-grade malignancy, including motility, invasiveness and a resistance to apoptosis; these can lead in turn to metastatic dissemination $(9,11)$. Moreover, during the process of EMT, epithelial cancer cells acquire molecular 
alterations that facilitate the loss of epithelial features and gain of mesenchymal phenotype. Such transformation promotes cancer cell migration and invasion (12-16). For this reason, increasing therapeutic substances or measures focusing on EMT have been investigated as potential therapies for preventing breast cancer metastasis.

Since more and more studies have demonstrated that herbal plant extracts exert antitumor effects, these herbal plant extracts have been considered as a practical approach to reduce the incidence of breast cancer. Moreover, since chemotherapeutics are limited and chemoresistance occurs frequently, these herbal plant extracts have also attracted increased attention in the exploration of effective antitumor drugs for breast cancer metastasis. Ferulic acid (4-hydroxy-3-methoxycinnamic acid), a widely distributed constituent of plants, was first isolated from Ferula foetida in 1866 (17). It has been described to act as a potent antioxidant by scavenging free radicals and enhancing the cell stress response through the upregulation of cytoprotective systems (18). Previous studies have indicated that ferulic acid could inhibit the expression and activity of cytotoxic enzymes, including inducible nitric oxide synthase, caspases and cyclooxygenase-2 (19). However, it also has been found that ferulic acid exhibits a potential treatment for many disorders, e.g. Alzheimer's disease (17), colon cancer (20), cardiovascular diseases (21), diabetes mellitus (22) and skin disease (23). Although it was reported that ferulic acidoctyl and -dodecyl esters, significantly blocked the growth of breast, lung, colon and central nervous system tumor cells with $\mathrm{IC}_{50}$ values ranging from 17.05 to $4.29 \mu \mathrm{g} / \mathrm{ml}$ for the breast and colon, respectively (24), the detailed mechanisms by which ferulic acid inhibits cell growth and metastasis of breast cancer have not been fully elucidated.

In the present study, we reported the anticancer activity of ferulic acid in vitro and in vivo. Our results indicate that ferulic acid inhibits breast cancer proliferation and induces apoptosis. We further demonstrated that ferulic acid suppresses breast cancer metastasis by regulating EMT. These data collectively suggest that ferulic acid may be a very promising candidate for breast cancer intervention and prevention.

\section{Materials and methods}

Reagents and cell lines. Ferulic acid $\left(\mathrm{C}_{10} \mathrm{H}_{10} \mathrm{O}_{4} ; \mathrm{MW}\right.$ : 194.18; shown in Fig. 1A) was purchased from Sigma Chemical Co. (Sigma, St. Louis, MO, USA). Human breast cancer cell line MDA-MB-231 was obtained from the American Type Culture Collection (ATCC; Manassas, VA, USA), and cultured in RPMI-1640 medium supplemented with $10 \%$ fetal bovine serum (FBS) and $1 \%$ penicillin/streptomycin. All the cells were incubated at $37^{\circ} \mathrm{C}$ in a humidified atmosphere containing $5 \% \mathrm{CO}_{2}$.

Cell viability assay. Cells seeded onto a 96-well plate at a density of $4 \times 10^{3}$ cells/well were treated with $100 \mu \mathrm{l}$ medium plus dimethyl sulfoxide (DMSO; vehicle control, final concentration $<0.1 \%$ ) or ferulic acid (final concentration: 3,10 , 30 and $100 \mu \mathrm{M}$ ), and incubated for 24,48 and $72 \mathrm{~h}$, respectively. At the end of the drug exposure duration, cell viability was assessed using the Cell Counting Kit-8 (CCK-8) assay according to the manufacturer's protocols (Dojindo, Gaithersburg, MD, USA). Six parallel replicates were measured for each sample. Each plate included a control well that contained medium only, which was used to obtain a background spectrometric absorbance value that was subtracted from the test sample readings. The data are expressed as the mean ratio of live cells (treated vs. control) \pm SD for 3 replicates.

Apoptosis analysis by flow cytometry. For apoptosis analysis, human breast cancer cell line MDA-MB-231 was treated with ferulic acid (final concentration: 10, 30 and $100 \mu \mathrm{M}$ ) and DMSO (vehicle control, final concentration $<0.1 \%$ ) for $48 \mathrm{~h}$, incubated with fluorescein isothiocyanate-Annexin $\mathrm{V}$ and propidium iodide (BD Biosciences) for $15 \mathrm{~min}$, and then analyzed by flow cytometry.

Caspase activity assay. Caspase-3 activity was measured using colorimetric assay kits (Beyotime Institute of Biotechnology, Jiangsu, China) according to the manufacturer's instructions. Briefly, after isolation by two-step collagenase digestion, the breast cancer cells were mixed with $50 \mu \mathrm{l}$ cold lysis buffer. The supernatant $(10 \mu \mathrm{l})$ was then mixed with $10 \mu \mathrm{l}$ caspase substrate and $80 \mu \mathrm{l}$ reaction buffer and incubated at $37^{\circ} \mathrm{C}$ for $2 \mathrm{~h}$ in the dark. The fluorescence intensity of the caspase substrate at $405 \mathrm{~nm}$ was measured using a microplate reader. The caspase activity was calculated as the OD405/100 $\mu \mathrm{g}$ protein.

Wound healing and Transwell migration assays. For the wound healing assay, we drew horizontal lines across the back of the wells of 6-well plates with a marker pen. The cells $\left(5 \times 10^{5}\right.$ cells/well) were plated into the 6 -well plates. On the following day, the confluent cell monolayers were carefully wounded (perpendicular to the horizontal lines) with sterile pipette tips and washed with phosphate-buffered saline (PBS) twice to remove cellular debris. Serum-free medium was added into the wells. Then, the wounded cell monolayers were cultured for another $48 \mathrm{~h}$ treat with or without different concentrations of ferulic acid. Images were captured under a microscope to observe the distribution of the cells at the scratch zone at the last time point. The degree of wound closure was then quantitatively evaluated using Image-Pro Plus software. Four fields from each well were documented, and each experiment was performed in triplicate.

A cell migration assay was performed using Transwell chambers with a pore size of $0.8 \mu \mathrm{m}$. Breast cancer cells were trypsinized, washed and suspended in medium without FBS. To the lower wells of the chambers, migration-inducing medium (with $10 \% \mathrm{FBS}$ ) was added. A total of $2 \times 10^{4}$ cells were seeded in serum-free medium in the upper chamber, containing various concentrations of ferulic acid (10, 30 and $100 \mu \mathrm{M}$ ) or vehicle solvent (DMSO). After incubating for $24 \mathrm{~h}$ at $37^{\circ} \mathrm{C}$, the cells in the upper chamber were carefully removed with a cotton swab, and the cells that had migrated to the reverse face of the membrane were fixed in methanol, stained with Giemsa solution and counted.

Immunofluorescence analysis. For the immunofluorescence experiments, cells were prepared and analyzed under a fluorescence microscope following the procedures previously described (25). Briefly, cells treated with or without 
ferulic acid were incubated with primary antibody against vimentin $(1: 100 ; 5741)$ or E-cadherin $(1: 200 ; 3195)$ (both from Cell Signaling Technology), and then incubated with DyLight 594 or 488 (CW Biotech, Beijing, China) secondary antibody against rabbit IgG. Cells were then counterstained with 4',6-diamidino-2-phenylindole (DAPI) (Roche, Basel, Switzerland) and imaged with the fluorescence microscope.

Western immunoblot analysis and antibodies. The cell lysate was prepared using a protein extraction kit (Active Motif Company, Carlsbad, CA, USA) according to the manufacturer's instructions. Protein concentrations were determined by BCA protein assay kit (Thermo Fisher Scientific, Inc., Waltham, MA, USA), protein was loaded onto $10-12 \%$ Tris-acrylamide gels, electrophoresed and then transferred to nitrocellulose membranes. After blocking non-specific binding sites, the membranes were probed with primary antibodies, including anti-E-cadherin $(1: 1,000 ; 3195)$, anti-claudin-1 (1:1,000; 13255), anti-N-cadherin $(1: 1,000 ; 4061)$, anti-vimentin $(1: 1,000 ; 5741)$, anti-Snail $(1: 1,000 ; 3897)$ (all from Cell Signaling Technology), anti-Slug (1:1,000; ab27568; Abcam) and anti- $\beta$-actin (1:1,000; sc47778; Santa Cruz Biotechnology). Labeled proteins were visualized by an ECL chemiluminescence kit (Millipore, Billerica, MA, USA).

RNA extraction and quantitative PCR ( $q P C R)$. MDA-MB-231 cells were treated with various concentrations of ferulic acid (3, $10,30$ and $100 \mu \mathrm{M})$. At $48 \mathrm{~h}$ after treatment, the total RNA was extracted using TRIzol reagent (Invitrogen), respectively. Reverse transcription reactions were performed using $2 \mu \mathrm{g}$ of total RNA and were subsequently processed using a reverse transcription kit (TransGen Biotech, Beijing, China). Specific products were amplified by qPCR using the following primers: E-cadherin, 5'-CGAGAGCTACACGTTCACGG-3' (forward) and 5'-GGGTGTCGAGGGAAAAATAGG-3' (reverse); claudin-1, 5'-CCTCCTGGGAGTGATAGCAAT-3' (forward) and 5'-GGCAACTAAAATAGCCAGACCT-3' (reverse); vimentin, 5'-GACGCCATCAACACCGAGTT-3' (forward) and 5'-CTTTGTCGTTGGTTAGCTGGT-3' (reverse); N-cadherin, 5'-AGCCAACCTTAACTGAGGAGT-3' (forward) and 5'-GGC AAGTTGATTGGAGGGATG-3' (reverse); Snail, 5'-TCGGAA GCCTAACTACAGCGA-3' (forward) and 5'-AGATGAGCATT GGCAGCGAG-3' (reverse); Slug, 5'-CGAACTGGACACACA TACAGTG-3' (forward) and 5'-CTGAGGATCTCTGGTTG TGGT-3' (reverse). Verification of the expression levels of the genes was performed by qPCR using TransStart Green qPCR SuperMix kit (TransGen Biotech).

Tissue processing, hematoxylin and eosin staining, and immunohistochemistry. Mouse tumors, lungs and liver were fixed with paraformaldehyde, embedded in paraffin, and then sectioned. Some sections were incubated with hematoxylin and eosin (H\&E), dehydrated and mounted. For immunohistochemical analysis, sections were boiled in retrieval solutions to expose the antigens, and incubated at $4^{\circ} \mathrm{C}$ overnight with anti-Ki67 (1:100; ab16667; Abcam) and anti-cleaved caspase-3 (1:300; 9661; Cell Signaling Technology). The section-affixed slides were counterstained with hematoxylin, dehydrated and mounted. The immunostaining results were independently evaluated by two pathologists.
Animal experiments. Five-week-old female BALB/c nude mice were purchased from the Institute of Laboratory Animal Science (Chinese Academy of Medical Science, Beijing, China). All procedures for the animal experiments were conducted according to the Animal Ethics Committee of Chongqing Medical University. All the animals were randomly divided into two groups of 9 mice each and housed according to the national and institutional guidelines for humane animal care. At 6 weeks of age, the mice were perorally (p.o.) gavaged with either $100 \mu \mathrm{l}$ ferulic acid (100 mg/kg weight) or normal saline (control) and the animals were gavaged daily during the experiment. At 7 weeks of age, the MDA-MB-231 cells were inoculated subcutaneously into the right flanks of the mice $\left(1.5 \times 10^{6}\right.$ cells/mouse). Body weights were monitored weekly as an indicator of overall health. Tumor diameter was measured every week, and tumor volumes were calculated with the formula: tumor volume $\left(\mathrm{mm}^{3}\right)=0.5 \mathrm{x}$ length $(\mathrm{mm}) \times$ width $^{2}\left(\mathrm{~mm}^{2}\right)$. The mice were sacrificed via $\mathrm{CO}_{2}$ asphyxiation 6 weeks after the transplant. Tumors were then removed, weighed and sent for immunohistochemistry (IHC) analysis.

For the tumor metastatic assay, six-week-old female BALB/c nude mice were p.o. gavaged with $100 \mu \mathrm{l}$ ferulic acid (100 mg/kg weight) or normal saline (control) for one week. Then, they were administered MDA-MB-231 cells ( $2.5 \times 10^{6}$ cells) via the tail vein (6 mice in each group), and the treatment continued until the end of the experiment. At the end of the experiment, on day 28 , the mice were sacrificed via $\mathrm{CO}_{2}$ asphyxiation. The lungs and livers were removed, and sent for H\&E staining.

Statistical analysis. Statistical analyses were performed using SPSS 13.0 software. Data from all the experiments are presented as means \pm SD and represent 3 independent experiments. One-way analysis of variance (ANOVA) or Student's t-test was used to compare means between treatment groups. A P-value of $<0.05$ was considered to indicate a statistically significant result.

\section{Results}

Effect offerulic acid on breast cancer proliferation and apoptosis. Breast cancer cell line MDA-MB-231 was treated with various concentrations of ferulic acid $(3,10,30$ and $100 \mu \mathrm{M})$. As shown in Fig. 1B, we observed that ferulic acid inhibited the cell proliferation of breast cancer cell line MDA-MB-231 in a dose-dependent manner. To further explore the effects of ferulic acid on cell apoptosis, MDA-MB-231 cells were used for the analysis of apoptosis by FACS. Following treatment with ferulic acid $(10,300$ and $100 \mu \mathrm{M})$ for $48 \mathrm{~h}$, significantly increased apoptosis was observed when breast cancer cells were treated with ferulic acid, which may partly contribute to the decreased cell viability (Fig. 2A and B). Moreover, the effect of ferulic acid on apoptosis was further confirmed by analyzing the activation of caspase-3 in the apoptosis pathway. In addition, the results showed that ferulic acid considerably enhanced the activity of caspase- 3 in breast cancer cells (Fig. 2C). Briefly, these data suggest that ferulic acid suppresses breast cancer cell proliferation and induces apoptosis. 
A<smiles>COc1cc(/C=C/C(=O)O)ccc1O</smiles>

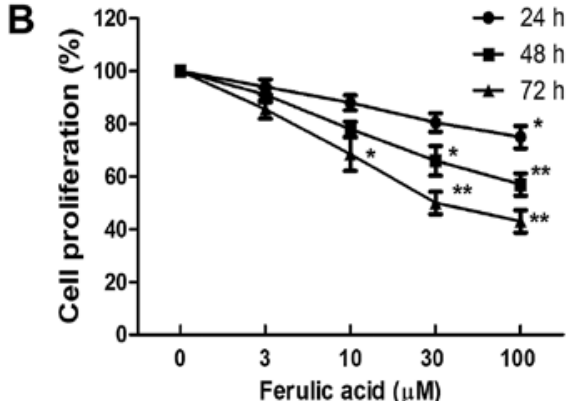

Figure 1. Ferulic acid inhibits cell proliferation in breast cancer cells. (A) Chemical structure of ferulic acid. (B) Breast cancer MDA-MB-231 cells were treated with various concentrations of ferulic acid for 24,48 and $72 \mathrm{~h}$, respectively. Cell viability was measured by CCK-8 kit. Data are expressed as ratios of treated cells to control cells. All the experiments were performed thrice in triplicate. Mean $\pm \mathrm{SD}$; ${ }^{*} \mathrm{p}<0.05$ and ${ }^{* *} \mathrm{p}<0.01$, vs. the control group.
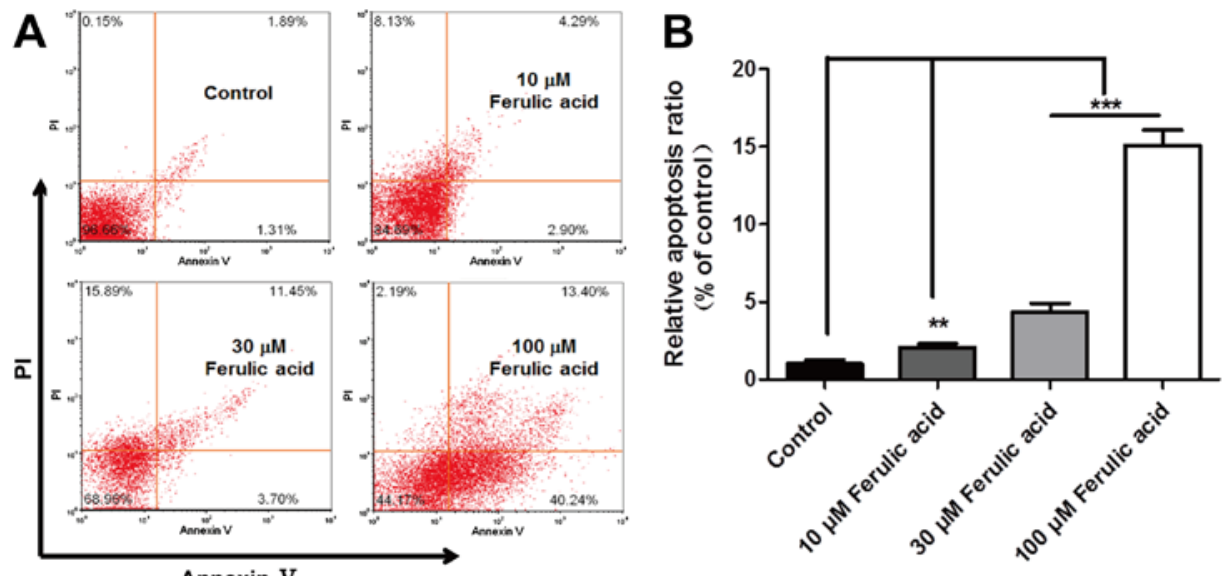

Annexin V

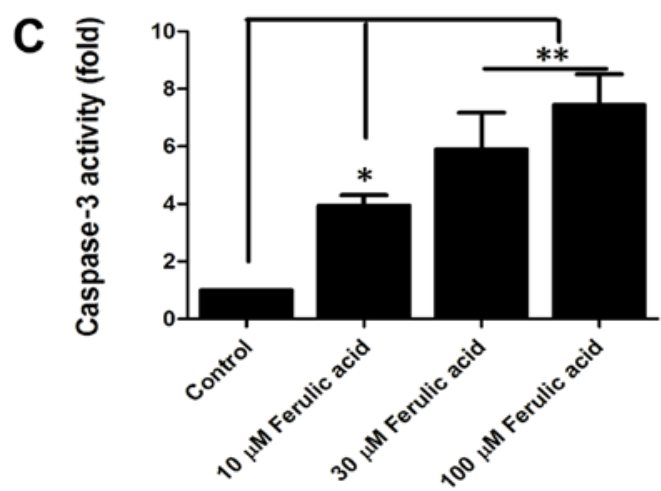

Figure 2. Ferulic acid induces cell apoptosis in breast cancer cells. (A) The apoptosis of human breast cancer MDA-MB-231 cells induced by ferulic acid was examined by flow cytometric analysis of Annexin V-FITC/PI staining. (B) Numbers inside dot plots indicate the percentages of apoptotic MDA-MB-231 cells (C) Caspase-3 activity was measured using colorimetric assay kits. The data are expressed as the absorbance at $405 \mathrm{~nm} / 100 \mu \mathrm{g}$ protein. All the experiments were performed thrice in triplicate. Mean $\pm \mathrm{SD} ;{ }^{*} \mathrm{p}<0.05,{ }^{* *} \mathrm{p}<0.01$ and ${ }^{* * *} \mathrm{p}<0.001$, vs. the control group.

Ferulic acid inhibits breast cancer cell migration. To investigate the role of ferulic acid in breast cancer metastasis, we investigated the migratory capacity of high metastatic cell line MDA-MB-231. We firstly carried out wound healing assays to evaluate the effects of ferulic acid on breast cancer cell migration at the concentrations of 10,30 and $100 \mu \mathrm{M}$. As shown in Fig. 3A and B, the migration across the wound edges was markedly slower after treatment with different concentrations of ferulic acid. From the Transwell assay, we also found that ferulic acid dose-dependently inhibited breast cancer cell migration (Fig. 3C and D). These results indicate that ferulic acid inhibits breast cancer migration even at a low concentra- tion $(10 \mu \mathrm{M})$ and that ferulic acid indeed possesses the ability to inhibit tumor metastasis in vitro.

Ferulic acid induces reversal of EMT. EMT is thought to be a driver of invasion and metastasis in different types of epithelial cancers (26). The most comprehensive theory describing how initially quiescent tumor cells acquire metastatic capability is EMT (27). A group of pleiotropic transcription factors (TFs) have been found capable of orchestrating EMT programs. Most EMT-TFs are transcriptional repressors and many, such as Snail, Slug, ZEB1 and Twist, directly repress mediators of epithelial adhesion, the most important of which 

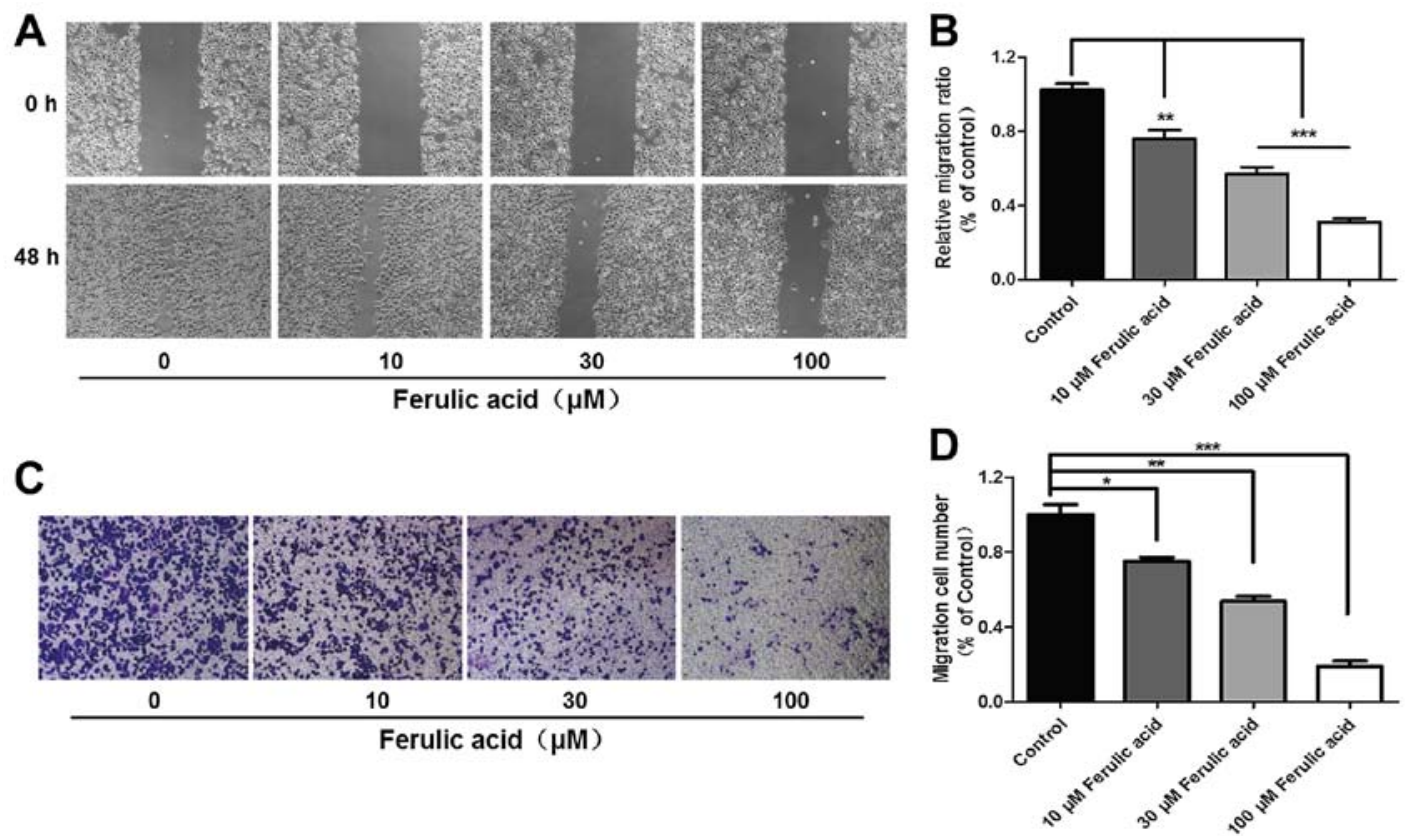

Figure 3. Ferulic acid inhibits breast cancer cell migration. (A and B) Ferulic acid inhibited MDA-MB-231 cell migration in a wound healing assay. Cells were wounded with a pipette and treated with different concentrations $(0,12.5,25$ and $50 \mu \mathrm{M})$ of ferulic acid. Microscopic observations were recorded 0 and $48 \mathrm{~h}$ after scratching the cell surface. A representative image from 3 independent experiments is shown. (C and D) Ferulic acid inhibited MDA-MB-231 cell migration in a Transwell assay. Images represented the cells that travelled through the micropore membrane and histograms show the percentage of migrant cells. All the experiments were performed thrice in triplicate. Mean $\pm \mathrm{SD} ;{ }^{*} \mathrm{p}<0.05,{ }^{* *} \mathrm{p}<0.01$ and ${ }^{* * *} \mathrm{p}<0.001$, vs. the control group.
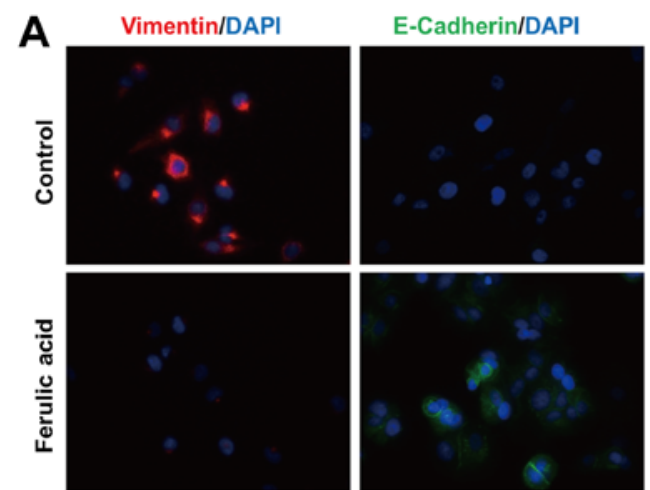

C

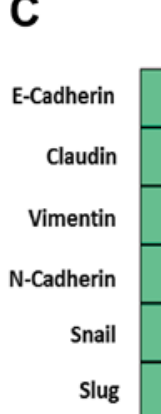

Ferulic acid

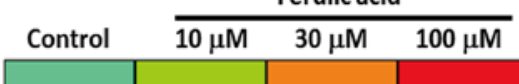

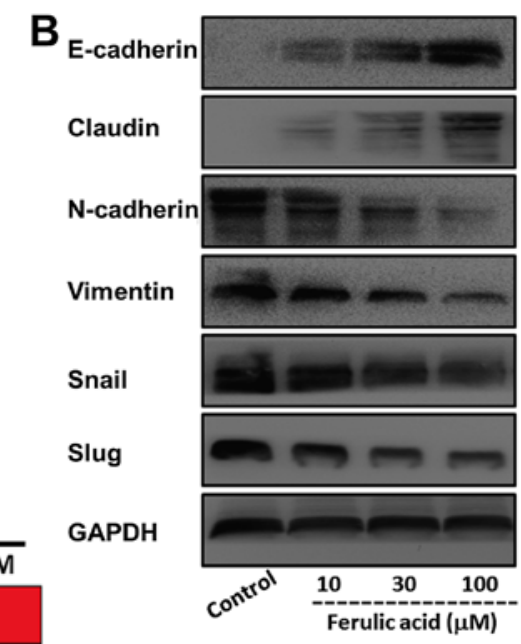

Figure 4. Reversal effect of ferulic acid on EMT in breast cancer cells. (A) Immunofluorescent staining shows the downregulation of vimentin and upregulation of E-cadherin in breast cancer cells treated with ferulic acid (30 $\mu \mathrm{M})$ for $48 \mathrm{~h}$ (original magnification, x400). Nuclei were stained with DAPI (blue), vimentin with DyLight 594 (red) and E-cadherin with DyLight 488 (green). Breast cancer cells were treated with different concentrations of ferulic acid for 48 h, and the effect of ferulic acid on the expression of EMT markers was then measured by western blotting (B) and quantitative PCR (C).

is E-cadherin, an integral component of adherens junctions and claudins, which are necessary for the assembly of tight junctions between adjacent cells (28-33). To evaluate the effect of ferulic acid on EMT, we treated mesenchymal-like breast cancer cell line MDA-MB-231 with ferulic acid at different concentrations $(10,30$ and $100 \mu \mathrm{M})$. We first detected the 

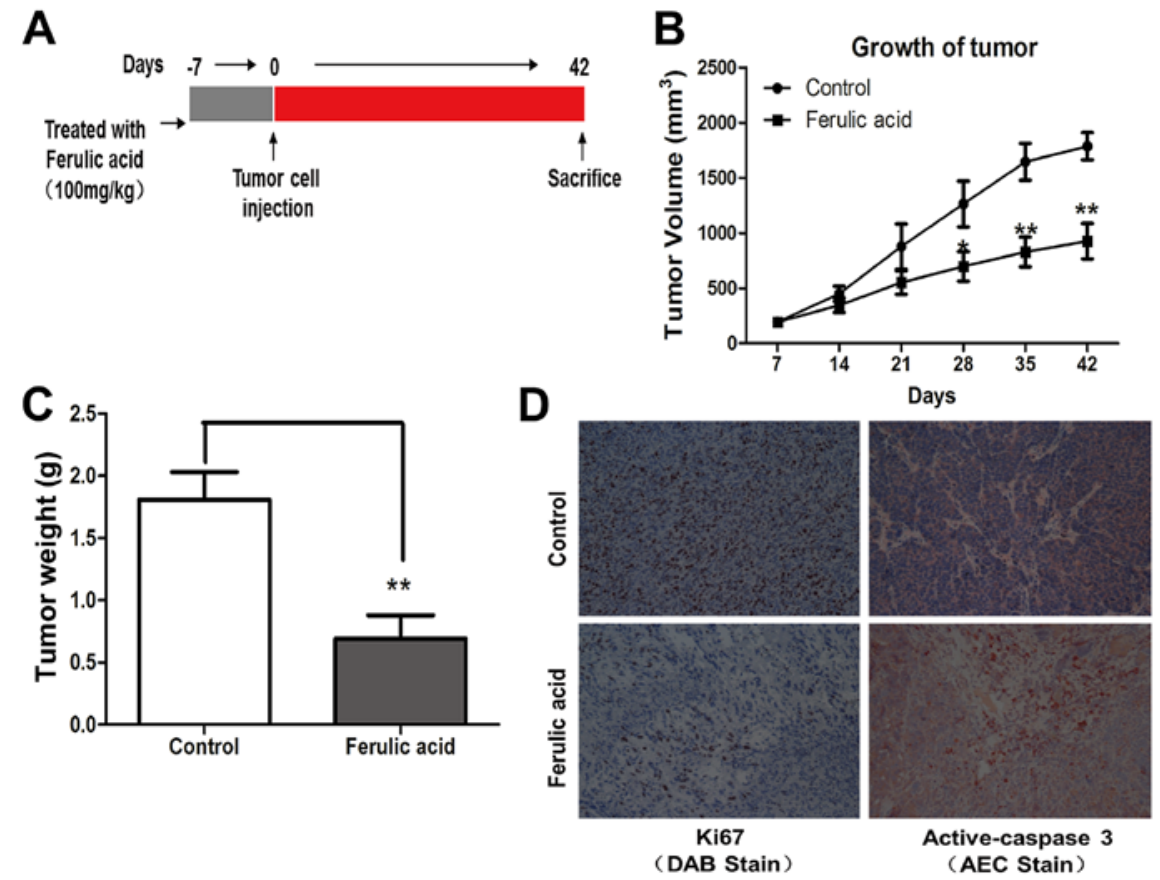

Figure 5. Antitumor activity of ferulic acid in an MDA-MB-231 breast cancer model. (A) Ferulic acid was orally initiated one week prior to tumor cell injection and continued during the experiment. A total of $1.5 \times 10^{6}$ breast cancer cells were respectively injected into the right flanks of nude mice ( $\mathrm{n}=9$ ). Tumor volume (B) and weight (C) were measured in the different groups. (D) Ki67 staining for cell proliferation and cleaved caspase-3 staining for apoptosis were evaluated by immunohistochemistry (original magnification, $\mathrm{x} 400$ ).

A

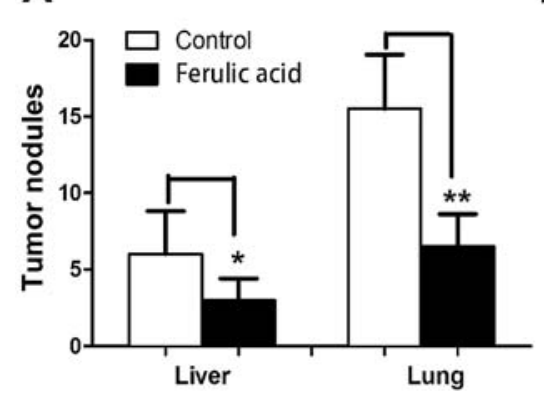

B

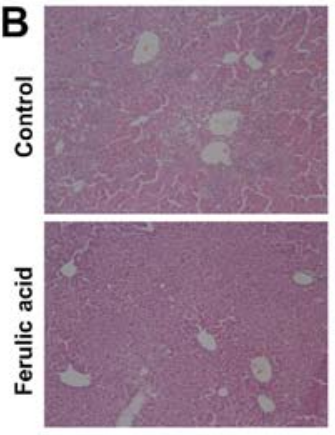

Liver

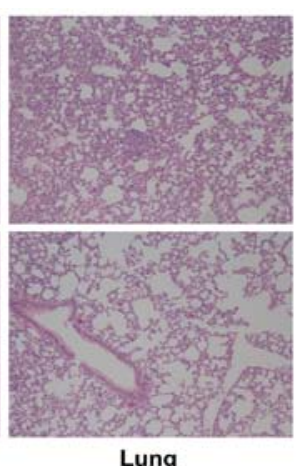

Figure 6. Ferulic acid suppresses the metastatic potential of breast cancer in vivo. (A) The number of tumor nodules that metastasized to the lungs and liver were analyzed. Ferulic acid decreased tumor metastasis both in lungs and liver. (B) Representative hematoxylin and eosin (H\&E) images of the tumor nodules in lungs and liver.

expression of E-cadherin and vimentin, a mesenchymal intermediary filament by immunofluorescence. As shown in Fig. 4A, the expression of vimentin was markedly decreased after treatment with ferulic acid. However, we observed a significant increase of E-cadherin. Moreover, we investigated the effects of ferulic acid on the expression of these EMT markers at protein and mRNA levels (Fig. 4B and C). Consistent with immunofluorescence, increased expression of epithelial markers and decreased mesenchymal markers were demonstrated in ferulic acid-treated cancer cells. Collectively, these observations support that ferulic acid induces an effective switch from a mesenchymal to an epithelial phenotype in breast cancer cells.

Ferulic acid exerts antitumor activities in vivo. Based on the in vitro findings described above, MDA-MB-231 xenograft model was employed to evaluate the antitumor potential of ferulic acid in vivo. Ferulic acid was orally initiated one week prior to tumor cell injection and then continued until the end of the experiment (Fig. 5A). Oral administration of ferulic acid had no detectable toxicity, as there were no differences in body weight between the control and treatment groups, and no signs of adverse health reactions, pain or distress were observed. As shown in Fig. 5B and C, the tumors observed from the xenografts treated with ferulic acid exhibited smaller tumor volumes and lower tumor weights as compared with the control mice. Consistent with the in vitro experiments, we detected a significant decrease in proliferation (Ki67 staining) and increase in apoptosis (active caspase-3 staining) in tumors from the ferulic acid-treated mice (Fig. 5D). These in vivo data were consistent with the in vitro results and confirmed that ferulic acid exerts a marked antitumor activity against breast cancer. 
Ferulic acid suppresses the metastatic potential of breast cancer in vivo. To investigate whether ferulic acid can affect breast cancer metastasis in vivo, we established a metastatic model by injecting human breast cancer MDA-MB-231 cells into the tail vein of $\mathrm{BALB} / \mathrm{c}$ nude mice. The metastasis of MDA-MB-231 cells into the lungs and liver was measured by $H \& E$ staining 4 weeks after injection. As shown in Fig. $6 \mathrm{~A}$ and $\mathrm{B}$, the tumor nodules on the surface of the lungs and liver were significantly decreased in the ferulic acid-treated mice $(100 \mathrm{mg} / \mathrm{kg}$, daily). In summary, our data demonstrated that ferulic acid significantly inhibits breast cancer metastasis in vivo.

\section{Discussion}

The standard treatment for breast cancer includes surgery, chemotherapy, hormonal therapy and radiotherapy. However, there are several 'bottle necks' for the treatment of breast cancer, and metastasis is one of the main causes leading to treatment failure. Since metastastic breast cancer patients have a poor prognosis and a high mortality rate, preventing and inhibiting the metastasis itself is crucial for controlling the disease, prolonging survival, and enhancing the patient quality of life. Chemotherapy is the main treatment strategy for metastatic breast cancer. Since effective therapeutic drugs are limited and drug resistance occurs frequently, to explore natural and alternative cancer treatments which can improve the treatment of metastatic breast cancer itself and mitigate the undesirable side-effects of its treatment becomes more and more important. In the present study, we found that ferulic acid, exhibited an antimigration effect in vitro and in vivo.

Numerous studies have shown that ferulic acid is an efficient scavenger of both reactive oxygen and nitrogen species (ROS and RNS, respectively), and several lines of evidence have also demonstrated that the cytoprotective effects of ferulic acid could be attributable to the downregulation of pathways involved in cell death and the upregulation of gene/ proteins which are able to enhance the cell stress response (34). It is widely accepted that carcinogenesis is a multi-stage and complex process, such as enhanced cell proliferation, chronic inflammation, free radical formation and the following damage to DNA, abnormal activation of proinflammatory pathways including cyclooxygenases and NOS. In addition, each of these events not only plays a key role in carcinogenesis per se, but also contributes to strengthening the toxic potential of the others, thus amplifying the cell proliferative cascade. In light of this, the ability of ferulic acid to scavenge free radicals, stimulate cytoprotective enzymes and inhibit cytotoxic systems account for the potential adjuvant role of ferulic acid in cancer therapy. Additionally, previous studies have reported the antineoplastic activity of ferulic acid in various types of cancers. For example, it was reported that ferulic acid exhibited antiproliferative effects on Caco-2 colon cancer cells by upregulating RABGAP1 and CEP2, which were involved in centrosome assembly as well as the gene for the $\mathrm{S}$ phase checkpoint protein SMC1L1 (20). Additionally, another study demonstrated that ferulic acid had significantly reduced plasma levels of lactic dehydrogenase and alkaline phosphatase in nicotine-treated rats by counteracting the nicotine-induced lipoperoxidation and DNA damage $(35,36)$. Although studies concerning the antitumor activity of ferulic acid are limited, a growing body of evidence supports the potentially important role in free radical-induced cancers. In the present study, we showed that ferulic acid effectively inhibited breast cancer cell proliferation and induced apoptosis, which support ferulic acid as an antitumor agent in breast cancer treatment.

EMT occurs during the early stages of the transformation of a tumor into a malignant neoplasm and is required for breast cancer metastasis (37). The concept that EMT involves the formation of metastatic cancer cells is based on the observation that acquisition of mesenchymal markers such as vimentin, Snail and Twist by epithelial carcinoma cells is associated with increased metastatic potential (38), as is nuclear overexpression of $\beta$-catenin (39) and loss of epithelial cell adhesion molecules such as E-cadherin (38-40). Moreover, accumulating evidence has demonstrated that EMT could result in cancer stem cell transformation, drug resistance and poorer prognosis for many types of human cancers. Therefore, pharmacologic inhibition of EMT or shutting down the function of EMT-TFs such as Twist, Snail and ZEB1 may be instrumental in cancer prevention and treatment $(41,42)$. In the present study, we verified the reversal effect of ferulic acid on EMT in metastatic breast cancer cell lines. Our results indicated that inhibition of EMT or inhibiting the function of EMT-TFs may be another mechanism involved in the antitumor activity of these herbal plant extracts.

Taken together, we first demonstrated the antitumor activity of ferulic acid in vitro and in vivo, and then we confirmed that ferulic acid could suppress breast cancer migration and metastasis by inhibiting the EMT process. These results strongly indicate the potential role of ferulic acid to inhibit both the growth and the metastasis of human breast cancer cells by mediating EMT. Furthermore, our findings support the conclusion that ferulic acid may be a potential candidate for metastastic breast cancer therapy.

\section{Acknowledgements}

The present study was supported by grants from the National Natural Science Foundation of China (no. 81472475).

\section{References}

1. Torre LA, Bray F, Siegel RL, Ferlay J, Lortet-Tieulent J and Jemal A: Global cancer statistics, 2012. CA Cancer J Clin 65: 87-108, 2015.

2. Hanahan D and Weinberg RA: Hallmarks of cancer: The next generation. Cell 144: 646-674, 2011.

3. Vanneman M and Dranoff G: Combining immunotherapy and targeted therapies in cancer treatment. Nat Rev Cancer 12: 237-251, 2012.

4. Nicolini A, Giardino R,Carpi A,FerrariP, Anselmi L, Colosimo S, Conte M, Fini M, Giavaresi G, Berti P, et al: Metastatic breast cancer: An updating. Biomed Pharmacother 60: 548-556, 2006.

5. Rubens RD: 7. Management of advanced breast cancer. Int J Clin Pract 55: 676-679, 2001

6. Yardley DA: Visceral disease in patients with metastatic breast cancer: Efficacy and safety of treatment with ixabepilone and other chemotherapeutic agents. Clin Breast Cancer 10: 64-73, 2010.

7. Cao H, Xu E, Liu H, Wan L and Lai M: Epithelial-mesenchymal transition in colorectal cancer metastasis: A system review. Pathol Res Pract 211: 557-569, 2015.

8. Nieto MA: The ins and outs of the epithelial to mesenchymal transition in health and disease. Annu Rev Cell Dev Biol 27: 347-376, 2011. 
9. Scheel C and Weinberg RA: Cancer stem cells and epithelialmesenchymal transition: Concepts and molecular links. Semin Cancer Biol 22: 396-403, 2012.

10. Shook D and Keller R: Mechanisms, mechanics and function of epithelial-mesenchymal transitions in early development. Mech Dev 120: 1351-1383, 2003.

11. Singh A and Settleman J: EMT, cancer stem cells and drug resistance: An emerging axis of evil in the war on cancer. Oncogene 29: 4741-4751, 2010.

12. Thiery JP and Lim CT: Tumor dissemination: An EMT affair Cancer Cell 23: 272-273, 2013.

13. Bill R and Christofori G: The relevance of EMT in breast cancer metastasis: Correlation or causality? FEBS Lett 589: 1577-1587, 2015.

14. Dalerba P, Cho RW and Clarke MF: Cancer stem cells: Models and concepts. Annu Rev Med 58: 267-284, 2007.

15. Armstrong AJ, Marengo MS, Oltean S, Kemeny G, Bitting RL, Turnbull JD, Herold CI, Marcom PK, George DJ and Garcia-Blanco MA: Circulating tumor cells from patients with advanced prostate and breast cancer display both epithelial and mesenchymal markers. Mol Cancer Res 9: 997-1007, 2011.

16. Wu Y, Sarkissyan M and Vadgama JV: Epithelial-mesenchymal transition and breast cancer. J Clin Med 5: pii: E13, 2016. doi: $10.3390 / \mathrm{jcm} 5020013$.

17. Sgarbossa A, Giacomazza D and di Carlo M: Ferulic Acid: A hope for Alzheimer's disease therapy from plants. Nutrients 7 : 5764-5782, 2015.

18. Kanski J, Aksenova M, Stoyanova A and Butterfield DA: Ferulic acid antioxidant protection against hydroxyl and peroxyl radical oxidation in synaptosomal and neuronal cell culture systems in vitro: Structure-activity studies. J Nutr Biochem 13: 273-281, 2002.

19. Mancuso C and Santangelo R: Ferulic acid: Pharmacological and toxicological aspects. Food Chem Toxicol 65: 185-195, 2014.

20. Janicke B, Hegardt C, Krogh M, Onning G, Akesson B, Cirenajwis HM and Oredsson SM: The antiproliferative effect of dietary fiber phenolic compounds ferulic acid and p-coumaric acid on the cell cycle of Caco-2 cells. Nutr Cancer 63: 611-622, 2011.

21. Alam MA, Sernia C and Brown L: Ferulic acid improves cardiovascular and kidney structure and function in hypertensive rats. J Cardiovasc Pharmacol 61: 240-249, 2013.

22. Roy S, Metya SK, Sannigrahi S, Rahaman N and Ahmed F: Treatment with ferulic acid to rats with streptozotocin-induced diabetes: Effects on oxidative stress, pro-inflammatory cytokines, and apoptosis in the pancreatic $\beta$ cell. Endocrine 44: 369-379, 2013.

23. Lin FH, Lin JY, Gupta RD, Tournas JA, Burch JA, Selim MA, Monteiro-Riviere NA, Grichnik JM, Zielinski J and Pinnell SR Ferulic acid stabilizes a solution of vitamins $C$ and $E$ and doubles its photoprotection of skin. J Invest Dermatol 125: 826-832, 2005 .

24. Jayaprakasam B, Vanisree M, Zhang Y, Dewitt DL and Nair MG: Impact of alkyl esters of caffeic and ferulic acids on tumor cell proliferation, cyclooxygenase enzyme, and lipid peroxidation. J Agric Food Chem 54: 5375-5381, 2006.

25. Cole L, Anderson M, Antin PB and Limesand SW: One process for pancreatic beta-cell coalescence into islets involves an epithelial-mesenchymal transition. J Endocrinol 203: 19-31, 2009.

26. Mani SA, Guo W, Liao MJ, Eaton EN, Ayyanan A, Zhou AY, Brooks M, Reinhard F, Zhang CC, Shipitsin M, et al: The epithelial-mesenchymal transition generates cells with properties of stem cells. Cell 133: 704-715, 2008 .

27. Gomes LR, Terra LF, Sogayar MC and Labriola L: Epithelialmesenchymal transition: Implications in cancer progression and metastasis. Curr Pharm Biotechnol 12: 1881-1890, 2011.
28. Cano A, Pérez-Moreno MA, Rodrigo I, Locascio A, Blanco MJ, del Barrio MG, Portillo F and Nieto MA: The transcription factor snail controls epithelial-mesenchymal transitions by repressing E-cadherin expression. Nat Cell Biol 2: 76-83, 2000.

29. Bolós V, Peinado H, Pérez-Moreno MA, Fraga MF, Esteller M and Cano A: The transcription factor Slug represses E-cadherin expression and induces epithelial to mesenchymal transitions: A comparison with Snail and E47 repressors. J Cell Sci 116 499-511, 2003

30. Eger A, Aigner K, Sonderegger S, Dampier B, Oehler S, Schreiber M, Berx G, Cano A, Beug H and Foisner R: DeltaEF1 is a transcriptional repressor of E-cadherin and regulates epithelial plasticity in breast cancer cells. Oncogene 24: 2375-2385, 2005.

31. Yang MH, Hsu DS, Wang HW, Wang HJ, Lan HY, Yang WH, Huang $\mathrm{CH}$, Kao SY, Tzeng CH, Tai SK, et al: Bmil is essential in Twistl-induced epithelial-mesenchymal transition. Nat Cell Biol 12: 982-992, 2010.

32. Ikenouchi J, Matsuda M, Furuse M and Tsukita S: Regulation of tight junctions during the epithelium-mesenchyme transition: Direct repression of the gene expression of claudins/occludin by Snail. J Cell Sci 116: 1959-1967, 2003.

33. Martínez-Estrada OM, Cullerés A, Soriano FX, Peinado $\mathrm{H}$, Bolós V, Martínez FO, Reina M, Cano A, Fabre M and Vilaró S: The transcription factors Slug and Snail act as repressors of Claudin-1 expression in epithelial cells. Biochem J 394: 449-457, 2006.

34. Barone E, Calabrese V and Mancuso C: Ferulic acid and its therapeutic potential as a hormetin for age-related diseases. Biogerontology 10: 97-108, 2009.

35. Adluri RS, Nagarajan D, Periyaswamy V and Venugopal PM: Dose-response effect of ferulic acid against nicotine-induced tissue damage and altered lipid levels in experimental rats: A pathohistological evaluation. Fundam Clin Pharmacol 22: 557-567, 2008.

36. Sudheer AR, Muthukumaran S, Kalpana C, Srinivasan M and Menon VP: Protective effect of ferulic acid on nicotine-induced DNA damage and cellular changes in cultured rat peripheral blood lymphocytes: A comparison with $N$-acetylcysteine. Toxicol In Vitro 21: 576-585, 2007.

37. Thiery JP, Acloque H, Huang RY and Nieto MA: Epithelialmesenchymal transitions in development and disease. Cell 139: 871-890, 2009

38. Thompson EW, Torri J, Sabol M, Sommers CL, Byers S, Valverius EM, Martin GR, Lippman ME, Stampfer MR and Dickson RB: Oncogene-induced basement membrane invasiveness in human mammary epithelial cells. Clin Exp Metastasis 12: 181-194, 1994.

39. Brabletz T, Jung A, Hermann K, Günther K, Hohenberger W and Kirchner T: Nuclear overexpression of the oncoprotein beta-catenin in colorectal cancer is localized predominantly at the invasion front. Pathol Res Pract 194: 701-704, 1998.

40. Xue C, Plieth D, Venkov C, Xu C and Neilson EG: The gatekeeper effect of epithelial-mesenchymal transition regulates the frequency of breast cancer metastasis. Cancer Res 63: 3386-3394, 2003.

41. Dave B, Mittal V, Tan N Toxicol In Vitro M and Chang JC: Epithelial-mesenchymal transition, cancer stem cells and treatment resistance. Breast Cancer Res 14: 202, 2012.

42. Foroni C, Broggini M, Generali D and Damia G: Epithelialmesenchymal transition and breast cancer: Role, molecular mechanisms and clinical impact. Cancer Treat Rev 38: 689-697, 2012. 